14

\title{
Адаптивная фильтрация физиологических артефактов на сигналах электроэнцефалограмм человека с использованием разложения по эмпирическим модам
}

\author{
(C) В.В. Грубов, А.Е. Руннова, А.Е. Храмов \\ Саратовский государственный технический университет им. Ю.А. Гагарина, \\ 410054 Саратов, Россия \\ e-mail: vvgrubov@gmail.com, hramovae@gmail.com
}

(Поступило в Редакцию 22 апреля 2017 г. В окончательной редакции 3 ноября 2017 г.)

\begin{abstract}
Предложен новый метод для адаптивной фильтрации экспериментальных сигналов электроэнцефалограмм человека и удаления различных типов физиологических артефактов. Алгоритм метода включает разложение сигнала электроэнцефалограммы по эмпирическим модам, определение числа эмпирических мод, принимаемых к рассмотрению, анализ эмпирических мод и поиск мод с артефактами, удаление этих мод и восстановление сигнала электроэнцефалограммы. Метод был протестирован на экспериментальных сигналах электроэнцефалограмм человека и показал высокую эффективность при удалении различных типов физиологических артефактов на электроэнцефалограммах.
\end{abstract}

DOI: 10.21883/JTF.2018.05.45908.2304

\section{Введение}

В настоящее время наблюдается значительный интерес к изучению колебательной активности в нейронных сетях головного мозга. Данная задача лежит на стыке многих областей науки: нейрофизиологии, медицины, биофизики, нелинейной динамики, математики и т. д. Основными источниками информации о работе головного мозга являются экспериментальные методы, в том числе запись различных сигналов мозга. Одним из наиболее распространенных и используемых в исследованиях сигналов головного мозга является электроэнцефалограмма (ЭЭГ), представляющая собой сумму электрических токов, генерируемых небольшой группой нейронов и регистрируемых при помощи электрода [1]. Поскольку нейронная сеть головного мозга является крайне сложной колебательной системой, сигнал ЭЭГ также имеет сложный спектральный состав, в котором выделяют ряд частотных диапазонов (дельта, альфа, бета, гамма и т.д.), различных характерных ритмов и осцилляторных паттернов, которые представляют интерес для исследователей как при изучении различных патологий (например, эпилепсии), так и при анализе различных функциональных проб и когнитивных процессов [2-5].

Задача анализа сложных сигналов и характерных осцилляторных паттернов на них традиционно относится к радиофизике и нелинейной динамике. В данной области разработано множество эффективных методов для исследования частотно-временно́го состава сигналов, например, оконное фурье-преобразование или непрерывный вейвлет-анализ [5]. Многие из этих методов оказываются весьма эффективными и при анализе сигналов ЭЭГ [6,7]. Тем не менее в большинстве случаев применение этих методов к исследованию сигналов ЭЭГ осложняется наличием на ней различных „паразитных“ процессов (паттернов) - шумов и так называемых артефактов. При записи ЭЭГ на результирующий сигнал оказывает влияние не только электрическая активность нейронного ансамбля головного мозга, но также внешние источники электрического сигнала и различные физиологические процессы, протекающие в организме. Шумы в сигнале ЭЭГ могут быть вызваны различными внешними источниками электрического сигнала, например, промышленной электрической сетью, накопленным статическим зарядом, плохим контактом регистрирующих электродов и т.д. Присутствие шумовых компонент в сигнале ЭЭГ можно значительно снизить путем обеспечения хорошего контакта электродов и экранирования регистрирующего оборудования. Артефакты на сигнале ЭЭГ обычно имеют физиологическую природу и чаще всего вызваны различными нестационарными процессами помимо мозговой активности, протекающими в организме при регистрации ЭЭГ. К таким процессам можно отнести движение глаз, кардиоритмы, активность лицевых и шейных мышц и т.д. [8,9].

Большинство артефактов на ЭЭГ имеют значительную амплитуду, которая во многих случаях превышает амплитуду несущей электрической активности ансамбля нейронов головного мозга. Кроме того, частотные диапазоны многих артефактов пересекаются с диапазонами паттернов, представляющих интерес для исследования. Например, активно исследуемые пик-волновые разряды, имеющие непосредственное отношение к проявлению абсанс-эпилепсии у человека, имеют основную частоту $3-4 \mathrm{~Hz}$ и соответствующую ей хорошо выраженную вторую гармонику с частотой $6-8 \mathrm{~Hz}$; сонные веретена, также изучаемые в рамках исследования нарушений сна и механизмов работы мозга, имеют частоту $12-14 \mathrm{~Hz}$. В то же время глазодвигательные артефакты, кардиоритмы и мышечная активность могут быть обнаружены на всем диапазоне $0.5-15 \mathrm{~Hz}$, который перекрывает три важных высокоинформативных низкочастотных диапазо- 
на ЭЭГ - дельта, тета и альфа [1-3]. Таким образом, присутствие артефактов и их сильная вариабельность значительно усложняют частотно-временно́й анализ сигналов ЭЭГ, что делает предварительную обработку и фильтрацию сигналов ЭЭГ важным этапом любых электроэнцефалографических исследований.

В настоящее время для фильтрации ЭЭГ от артефактов применяют ряд различных методов. Одним из наиболее простых и часто используемых является метод на основе визуального поиска артефактов [10,11]. Метод предполагает визуальный (или полуавтоматический) анализ временны́х реализаций ЭЭГ и поиск артефактов опытным нейрофизиологом. Обнаруженные артефакты удаляются из сигнала вручную или автоматически. Следует отметить, что данный метод требует экспертного знания структуры сигналов ЭЭГ и значительных затрат времени, особенно при обработке продолжительных записей ЭЭГ. Кроме того, основным способом удаления артефактов в данном случае является полное удаление отрезка ЭЭГ, содержащего артефакт, или обнуление амплитуды сигнала на этом отрезке. Такая процедура неизбежно приводит к искажению сигнала ЭЭГ или полной потере информации о частотно-временно́й структуре сигнала на данном отрезке [12]. Сокращение количества данных ЭЭГ для исследования критично в условиях ограниченного объема выборки данных. Например, 10-минутная запись ЭЭГ здорового человека после удаления артефактов описанным методом может сократиться до 2-3 min. Сокращение записи ЭЭГ для исследования снижает эффективность диагностики, а также увеличивает стоимость проведения экспериментальных работ.

Другие методы по удалению артефактов основаны на различных методах разложения и преобразования сигналов, например, на анализе независимых компонент $[4,13,14]$, регрессионном анализе [15], преобразовании Грамма-Шмидта [16]. Данные методы обладают достаточно высокой точностью выделения артефактов и малым искажением структуры сигналов ЭЭГ. Однако алгоритмы большинства подобных методов основаны на совместном анализе ЭЭГ и других физиологических сигналов, содержащих информацию об определенных типах артефактов - например, электроокулограмма (ЭОГ) содержит глазодвигательные артефакты, электрокардиограмма (ЭКГ) содержит кардиоритмы и т.д. Для использования этих методов необходимо обеспечить запись дополнительных сигналов, что не всегда возможно в силу отсутствия необходимого оборудования или исследования ранее записанных сигналов. Также применение данных методов ограничено возможностью удаления лишь некоторых определенных типов артефактов (например, глазодвигательных в случае с преобразованием Грамма-Шмидта).

Таким образом, важной задачей является разработка методов фильтрации сигналов ЭЭГ, не искажающих структуру сигнала ЭЭГ и в то же время не требующих записи дополнительных физиологических сигналов.
Одним из перспективных методов для обработки сигнала ЭЭГ и удаления физиологических артефактов является разложение сигнала по эмпирическим модам $[17,18]$. Уже были предприняты попытки разработать методы с использованием эмпирических мод, например, для удаления глазодвигательных артефактов [19], а также для выделения кортикальных паттернов бетаактивности на ЭЭГ [20]. Данные работы продемонстрировали перспективность разложения по эмпирическим модам, однако полученные результаты лежат в достаточно узкой области - удаление артефактов только конкретного типа (глазодвигательных) или выделение весьма специфического типа активности на ЭЭГ.

В настоящей работе было проведено обобщение подхода к фильтрации сигналов ЭЭГ на основе эмпирических мод и предложен новый метод удаления широкого спектра физиологических артефактов. Предложенный метод не требует регистрации дополнительных физиологических сигналов (ЭОГ, ЭКГ, МЭГ), что отличает его от большинства классических методов удаления артефактов, а также предлагает простой и унифицированный алгоритм фильтрации ЭЭГ, структура которого не зависит от типа удаляемых артефактов, но в то же время позволяет удалять артефакты различных типов, в отличие от подходов в работах $[19,20]$.

\section{1. Разложение сигнала по эмпирическим модам}

Разложение сигнала по эмпирическим модам является частью преобразования Гильберта-Хуанга и представляет собой один из современных методов частотно-временно́го анализа нелинейных нестационарных сигналов [17-21]. Данный метод позволяет представить исследуемый сигнал в виде набора амплитудномодулированных компонент с нулевым средним - так называемых эмпирических мод.

При анализе экспериментальных данных одним из этапов предварительной обработки является сведение сигнала к нулевому среднему уровню, что позволяет избавиться от проблем, связанных с некорректным определением мгновенной частоты эмпирических мод. Однако это не всегда выполняется для нестационарных сигналов (например, ЭЭГ), среднее значение которых может изменяться во времени и отличаться от нуля на локальных участках [22,23]. Для корректного определения эмпирических мод и их мгновенных частот в рамках метода предлагается выполнение следующих условий:

1. Локальное среднее значение каждой эмпирической моды должно быть равно нулю.

2. Число пересечений нулевого уровня графиком каждой эмпирической моды и число локальных максимумов (или минимумов) этой моды должны совпадать или отличаться не более чем на единицу.

Процедура разложения исследуемого сигнала $x(t)$ по эмпирическим модам предполагает следующий алгоритм: 
1. Нахождение всех экстремумов (минимумов и максимумов) исследуемого сигнала $x(t)$.

2. Интерполяция сигнала между минимумами и максимумами и построение двух соответствующих огибающих: $e_{\min }(t)$ и $e_{\max }(t)$.

3. Расчет низкочастотной составляющей сигнала (тренда) как среднего между двумя огибающими:

$$
m_{1}(t)=\frac{e_{\min }(t)+e_{\max }(t)}{2} .
$$

4. Расчет высокочастотной составляющей сигнала как разницы исходного сигнала и тренда: $d_{1}(t)=x(t)-m_{1}(t)$.

Если для $d_{1}(t)$ выполняются описанные выше условия, то $d_{1}(t)$ можно считать первой эмпирической модой $c_{1}(t)$ рассматриваемого сигнала, т. е. $c_{1}(t)=d_{1}(t)$.

В противном случае, если среднее значение $d_{1}(t)$ отлично от нуля, необходимо выполнение итерационной процедуры:

$$
\begin{gathered}
d_{11}(t)=d_{1}(t)-m_{11}(t), \\
d_{12}(t)=d_{11}(t)-m_{12}(t), \\
\cdots \\
d_{1 k}(t)=d_{1(k-1)}(t)-m_{1 k}(t),
\end{gathered}
$$

где $m_{1 k}(t)$ - тренд, рассчитанный для сигнала $d_{1(k-1)}(t)$. Итерационная процедура заканчивается, когда $d_{1 k}(t)$ становится сигналом с нулевым средним. В данном случае $d_{1 k}(t)$ будет являться первой эмпирической модой, т. е. $c_{1}(t)=d_{1 k}(t)$.

5. Вычитание первой эмпирической моды $c_{1}(t)$ из исходного сигнала $x(t)$ и получение остатка $r_{1}(t)$ : $r_{1}(t)=x(t)-c_{1}(t)$.

Для нахождения второй эмпирической моды действия, описанные в п. 1-4, повторяются для сигнала $r_{1}(t)$ вместо исходного сигнала $x(t)$, в ходе данной процедуры получается новое значение $r_{2}(t)$, которое используется для нахождения третьей эмпирической моды и т.д.

Пример работы алгоритма и процесс получения первой эмпирической моды показаны на рис. 1, где приведены исходный тестовых сигнал человеческой ЭЭГ из затылочной области мозга (электрод О1 в случае международной схемы расстановки „10-20“ регистрирующих ЭЭГ электродов) (рис. 1,a), и результаты последовательных шагов алгоритма: рис. $1, b$ - экстремумы на рассматриваемом сигнале, рис. $1, c$ - две огибающие по максимумам и минимумам, рис. $1, d-$ низкочастотная составляющая (тренд) сигнала, рис. $1, e-$ высокочастотная составляющая сигнала (эмпирическая мода). На рис. $1, b-e$ исходный сигнал обозначен серым, результаты каждого из шагов алгоритма - черным.

С точки зрения частотно-временно́го анализа разложение по эмпирическим модам существенно отличается от большинства методов анализа сигналов. Базисные функции, по которым производится разложение, в данном случае не заданы заранее (как, например, в Фурье или вейвлет-анализе), а определяются в ходе разложения

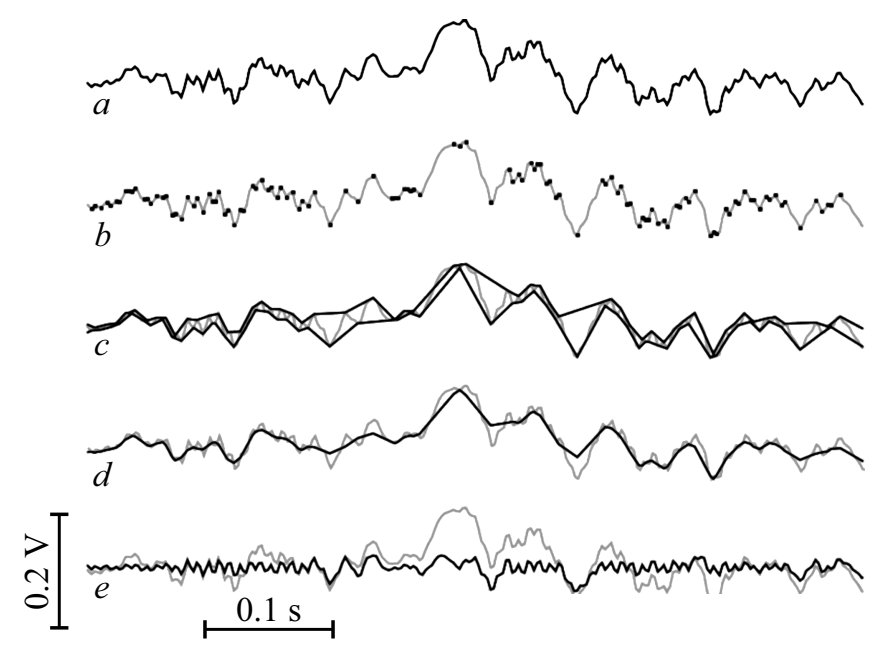

Рис. 1. Пошаговая демонстрация процедуры нахождения первой эмпирической моды: исходный сигнал ЭЭГ $(a)$, найденные экстремумы сигнала (отмечены точками) $(b)$, две огибающие, построенные по максимумам и минимумам $(c)$, рассчитанный тренд сигнала $(d)$, рассчитанная эмпирическая мода $(e)$.

из самого исследуемого сигнала. Общее количество эмпирических мод и частотно-временны́е характеристики каждой отдельной моды напрямую зависят от исследуемого сигнала. Это свойство делает разложение по эмпирическим модам высокоадаптивным инструментом анализа сигналов. Первая эмпирическая мода в разложении имеет самую высокую частоту, а с ростом порядкового номера эмпирической моды снижается значение ее основной частоты. Исследования показывают [24,25], что во многих случаях различные эмпирические моды по своему частотному составу соответствуют различным характерным осцилляторным паттернам на сигналах ЭЭГ. Таким образом, частотно-временно́й анализ и выделение конкретных осцилляторных паттернов (включая артефакты) могут быть сведены к анализу одной или нескольких эмпирических мод сигнала ЭЭГ.

Данное свойство разложения по эмпирическим модам проиллюстрировано на рис. 2, на котором приведен экспериментальный сигнал ЭЭГ человека, снятый с лобного электрода Fp1 (рис. 2,a) в случае стандартной схемы расстановки „10-20“, на котором присутствуют несколько глазодвигательных артефактов, а также первые три эмпирические моды для рассматриваемого сигнала ЭЭГ (рис. 2, $b-d$ ). Дополнительно на рис. 2 представлены вейвлет-спектры, построенные с базисным Морле-вейвлетом с центральной частотой $2 \pi$ [5], для исходного сигнала ЭЭГ и трех первых эмпирических мод. В данном случае вейвлет-анализ выступает не в роли самостоятельного инструмента для анализа сигналов, а используется лишь для наглядной иллюстрации частотно-временно́й структуры сигнала. Глазодвигательные артефакты на рис. 2 обозначены затененными серыми полосами. Данные артефакты представляют собой короткие $(\sim 300 \mathrm{~ms})$ осцилляторные паттерны с высокой амплитудой $1-1.5 \mathrm{~V}$. Из вейвлет-спетра на рис. 2, $a$ 
$a$
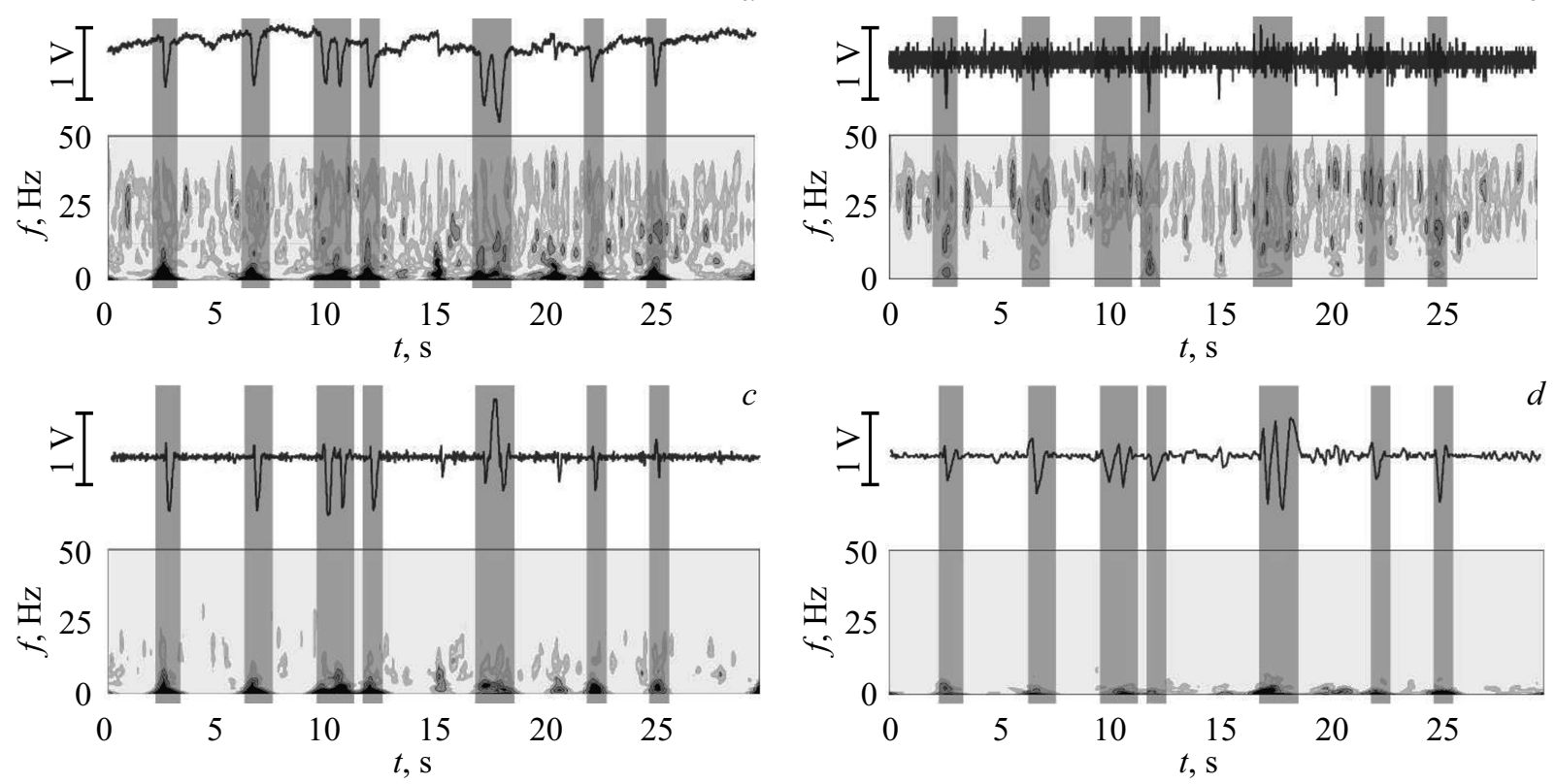

Рис. 2. Пример разложения по эмпирическим модам: сигнал ЭЭГ с несколькими глазодвигательными артефактами (a) и первые три эмпирические моды для него $(b-d)$; для каждого из сигналов приведен вейвлетный спектр, который иллюстрирует частотновременну́ю структуру сигнала; артефакты обозначены затененными рамками.

видно, что исходный сигнал ЭЭГ содержит различные ритмы и паттерны в широком диапазоне $0.5-50 \mathrm{~Hz}$, в то время как глазодвигательные артефакты возникают в диапазоне $0.5-5 \mathrm{~Hz}$. Вейвлетный спектр первой эмпирической моды (рис. 2,b) демонстрирует самые высокие частоты, как следует из процедуры разложения по эмпирическим модам, и потому данная мода содержит высокочастотные и информативные компоненты сигнала ЭЭГ. Рис. $2, c, d$ содержит вторую и третью эмпирические моды сигнала ЭЭГ вместе с их вейвлетными спектрами. Данные спектры в основном состоят из низких $(\sim 0.5-5 \mathrm{~Hz})$ частот, которые соответствуют фоновой ЭЭГ активности и диапазону глазодвигательных артефактов. Таким образом, в данном случае глазодвигательные артефакты могут быть локализованы во второй и третьей эмпирических модах, в то время как первая эмпирическая мода соответствует сигналу ЭЭГ, очищенному от артефактов. Данная процедура локализации артефактов на ЭЭГ при помощи эмпирических мод была расширена для выделения артефактов других типов и была использована в качестве ключевого элемента при разработке нового метода фильтрации сигналов ЭЭГ.

\section{2. Метод удаления артефактов на сигналах ЭЭГ}

В данной работе предложен новый метод для фильтрации сигналов ЭЭГ от артефактов на базе разложения по эмпирическим модам. Алгоритм разработанного метода имеет следующий вид:
1. Разложение исследуемого сигнала ЭЭГ на набор эмпирических мод.

2. Нахождение эмпирических мод, содержащих физиологические артефакты.

3. Удаление эмпирических мод, содержащих физиологические артефакты.

4. Восстановление сигнала ЭЭГ из оставшихся эмпирических мод.

На первом этапе алгоритма производится разложение сигнала ЭЭГ по эмпирическим модам, а также определяется общее количество эмпирических мод, принимаемых к рассмотрению.

Нужно отметить, что в ходе разложения сигнала ЭЭГ каждая эмпирическая мода оказывается короче исходного сигнала и предыдущей моды. Это происходит на этапе расчета тренда сигнала, поскольку из-за неравного количества минимумов и максимумов в сигнале одна из огибающих $e_{\min }(t)$ или $e_{\max }(t)$ оказывается короче другой, и часть точек из другой огибающей необходимо удалить для возможности расчета тренда по формуле $m(t)=\frac{e_{\min }(t)+e_{\max }(t)}{2}$. В то же время чем выше порядковый номер эмпирической моды, тем ниже ее основная частота, что приводит к тому, что высшие эмпирические моды имеют крайне низкие частоты. Первые несколько эмпирических мод теряют лишь незначительное количество точек по сравнению с исходным сигналом, однако с ростом порядкового номера моды потери становятся более заметными.

Данная особенность эмпирических мод проиллюстрирована на рис. 3, где приведен типичный сигнал ЭЭГ (рис. 3,a), его первая эмпирическая мода (рис. 3,b) 


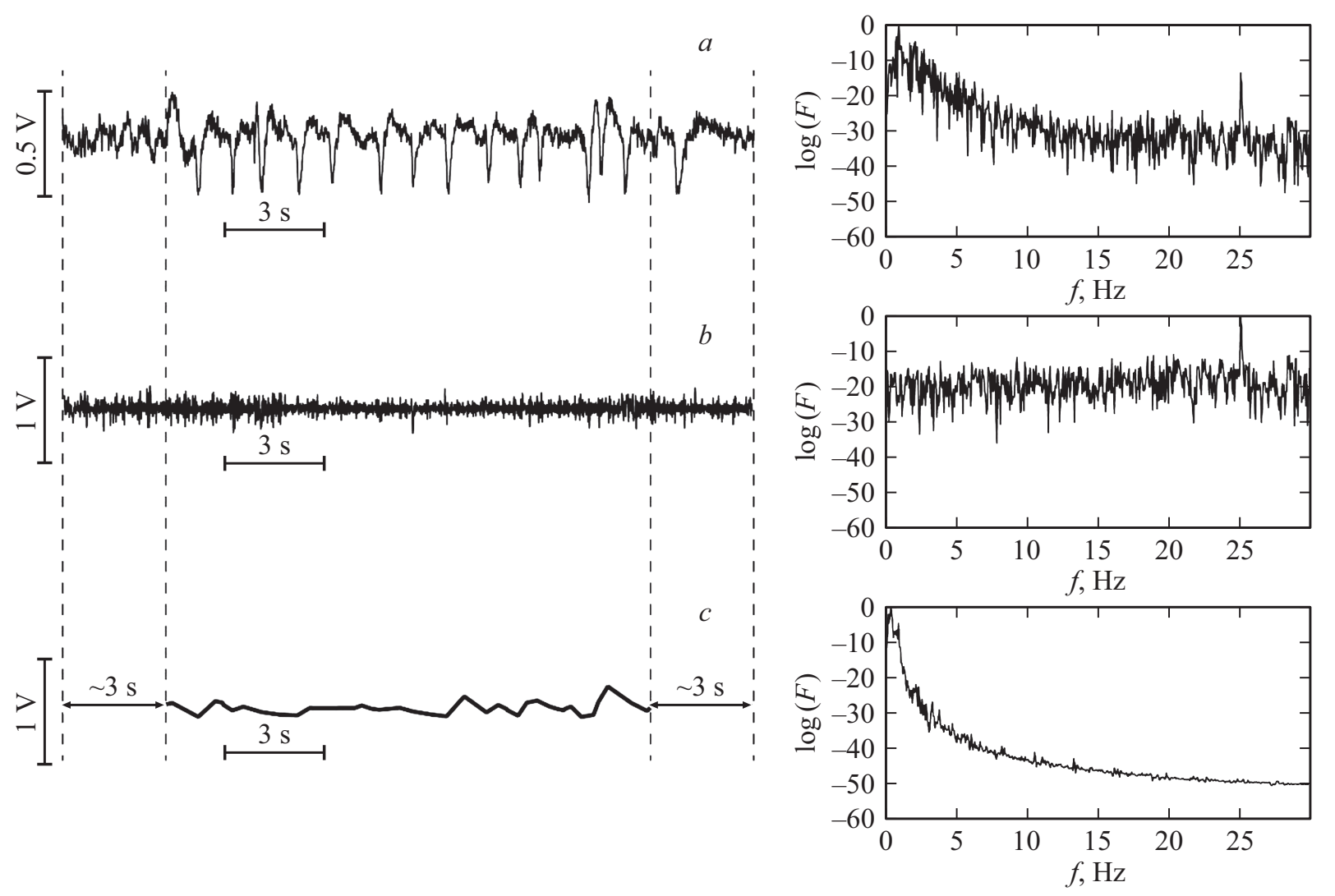

Рис. 3. Пример сравнения характеристик младших и старших эмпирических мод: сигнал ЭЭГ (a), первая эмпирическая мода $(b)$ и пятая эмпирическая мода $(c)$; для каждого из сигналов приведен фурье-спектр в логарифмическом масштабе, который иллюстрирует частотный состав сигнала.

и пятая эмпирическая мода (рис. 3,c). Дополнительно на рис. 3 приведены фурье-спектры в логарифмическом масштабе для сигнала ЭЭГ и его эмпирических мод. Из рис. $3, b$ видно, что первая эмпирическая мода содержит различные частоты с пиком в районе 3 и $25 \mathrm{~Hz}$, а длина сигнала в данном случае практически не отличается от длины исходного сигнала ЭЭГ. Пятая эмпирическая мода (одна из старших), представленная на pис. 3, $c$, содержит, как видно из ее фурье-спектра, низкие частоты $\sim 0.5 \mathrm{~Hz}$, которые соответствуют медленноволновой активности и различным шумам на ЭЭГ, которые обычно не анализируются ни в клинической практике, ни при проведении когнитивных исследований, а значит данная мода не содержит полезной информации. Отметим также, что пятая эмпирическая мода оказывается короче, чем исходный сигнал ЭЭГ. Такая разница может составлять до нескольких секунд, что не критично для длительных записей ЭЭГ, но может играть важную роль при анализе коротких интервалов ЭЭГ или при анализе процессов в головном мозге в режиме реального времени (например, при создании интерфейсов мозгкомпьютер) [26,27].

Особенность алгоритма предлагаемого метода заключается в том, что восстановление сигнала ЭЭГ на последнем этапе алгоритма производится путем суммирования всех оставшихся эмпирических мод, а следо- вательно, восстановленный сигнал будет иметь длину самой короткой эмпирической моды из этой суммы. Таким образом, в ходе анализа необходимо подобрать оптимальное число эмпирических мод для рассмотрения, чтобы, с одной стороны, учесть максимальное количество мод, содержащих полезную информацию, а с другой стороны, не взять слишком много старших мод, что привело бы к существенному сокращению длины фильтрованного сигнала за счет краевых эффектов. Исследования показывают, что в случае сигналов ЭЭГ человека частоты ниже $0.5-1 \mathrm{~Hz}$ относятся в основном к фоновой активности и шумам (рис. 2,c). Таким образом, в алгоритме предложенного метода к рассмотрению берутся только эмпирические моды с главной частотой $f_{m}>0.5 \mathrm{~Hz}(m=1,2, \ldots, M-$ порядковый номер эмпирической моды) и общее число рассматриваемых мод равняется $M$. При регистрации ЭЭГ с частотой дискретизации $250 \mathrm{~Hz}$ число анализируемых эмпирических мод обычно не превышает значения 5.

На втором этапе алгоритма среди рассматриваемых эмпирических мод производится поиск мод, содержащих физиологические артефакты. Данная процедура проводится при помощи непрерывного вейвлетпреобразования. Для этого на исходном сигнале ЭЭГ и всех эмпирических модах выбирается один и тот же небольшой отрезок, содержащий один или несколько 

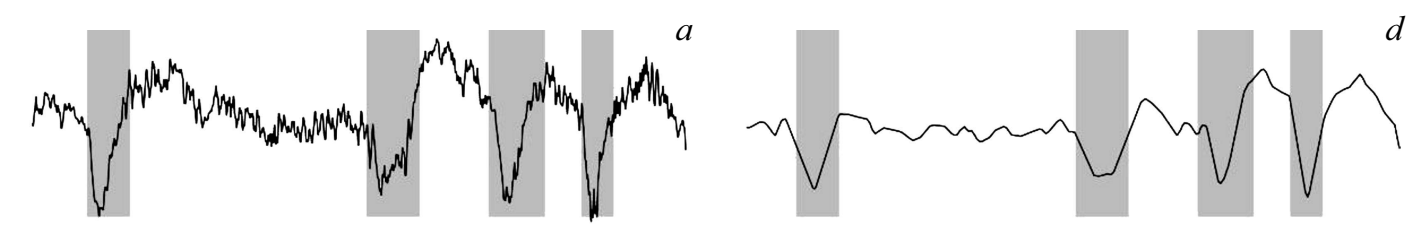

$b$
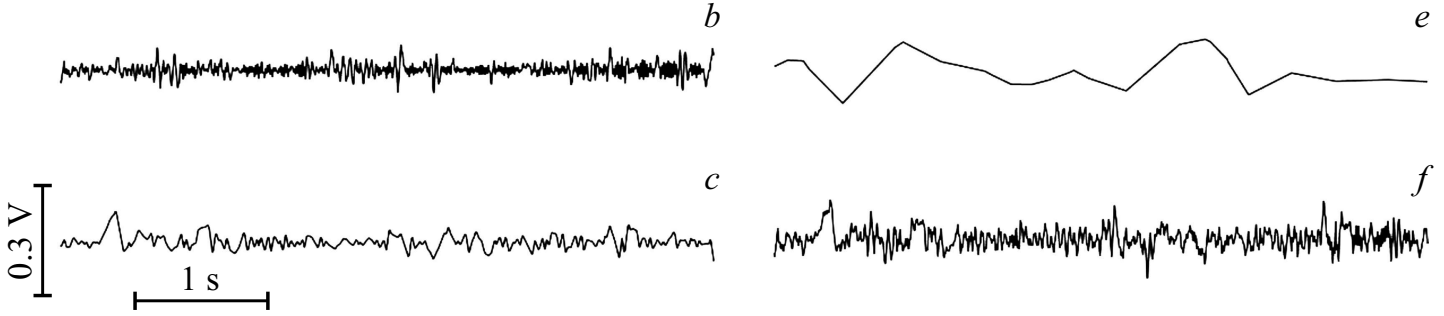

Рис. 4. Пример работы метода для фильтрации сигналов ЭЭГ от артефактов: сигнал ЭЭГ с несколькими глазодвигательными артефактами (выделены затененными рамками) (a), четыре эмпирических моды сигнала ЭЭГ $(b-e)$ и восстановленный сигнал ЭЭГ $(f)$.

интересующих артефактов. Далее для этого отрезка на ЭЭГ и эмпирических модах строятся соответствующие вейвлет-спектры и производится их анализ. Из клинической практики и исследований ЭЭГ известно, что большая часть физиологических артефактов обладает специфическими частотно-временны́ми характеристиками, такими как частотный диапазон, главная частота, продолжительность, волновая форма и т.д. Данные свойства в совокупности создают характерный образ на вейвлет-спектре для каждого типа артефакта. Например, в случае с глазодвигательными артефактами на вейвлетном спектре наблюдается резкое увеличение вейвлетной энергии в частотном диапазоне $0.5-5 \mathrm{~Hz}$ на протяжении $\sim 300-500 \mathrm{~ms}$ (рис. 2). В предложенном методе образы артефактов сначала определяются на вейвлетном спектре исходного сигнала ЭЭГ, а затем анализируются вейвлетные спектры каждой отдельной эмпирической моды. Считается, что эмпирическая мода содержит искомый тип артефактов, если на вейвлетном спектре этой моды присутствует образ данного артефакта.

На третьем этапе алгоритма все эмпирические моды, на которых было обнаружено присутствие артефактов, удаляются из рассмотрения. На четвертом этапе производится восстановление сигнала ЭЭГ. Для этого оставшиеся эмпирические моды, не содержащие артефакты, суммируются:

$$
U(t)=\sum_{i=1}^{N, i \neq n_{1}, n_{2} \ldots} M_{i}(t),
$$

где $U(t)$ - восстановленный сигнал ЭЭГ, $M_{i}(t)-$ эмпирические моды, $i$ - номер текущей моды, $N-$ общее число эмпирических мод, $n_{1}, n_{2} \ldots$ - номера эмпирических мод, содержащих артефакты.

Результатом работы предложенного метода является восстановленный сигнал ЭЭГ, на котором удалены физиологические артефакты.
Пример работы метода проиллюстрирован на рис. 4 и 5. На рис. 4 приведен небольшой отрезок сигнала ЭЭГ с канала T4, содержащий несколько глазодвигательных артефактов (выделены затененными рамками) (рис. 4, a), четыре эмпирические моды сигнала ЭЭГ (рис. 4, $b-e$ ), рассчитанные на первом шаге алгоритма метода, а также результат фильтрации - сигнал ЭЭГ, восстановленный после суммирования эмпирических мод (рис. $4, f$ ). Рис. 5 содержит вейвлетные спектры, соответствующие сигналам на рис. 4. Из рис. $4, b-d$ и соответствующих вейвлетных спектров на рис. $5, b-d$ видно, что первые три эмпирические моды содержат в основном высокие частоты, в то время как четвертая эмпирическая мода (рис. 4,e, рис. 5,e) имеет частоту ниже $1 \mathrm{~Hz}$. Таким образом, в данном случае к рассмотрению принимаются только первые три эмпирические моды. Далее в соответствии со вторым шагом алгоритма производится локализация артефактов на одной из мод. На вейвлетном спектре на рис. 5, $a$ затененной рамкой отмечен образ рассматриваемых артефактов, такой же образ встречается на вейвлетном спектре третьей эмпирической моды на рис. $5, d$. В данном случае артефакты были обнаружены на третьей эмпирической моде, которая удаляется в соответствии с третьим шагом алгоритма. После этого остаются только первая и вторая эмпирические моды, которые суммируются на четвертом шаге алгоритма, в результате чего получается восстановленный сигнал ЭЭГ (рис. 4,f). Из рис. 5, $f$ видно, что вейвлетный спектр восстановленного сигнала соответствует спектру исходного сигнала ЭЭГ (рис. 5, $a$ ), однако не содержит образов рассматриваемых артефактов.

Несмотря на то что описанный выше пример приведен для удаления глазодвигательных артефактов, разработанный метод может быть также применен для удаления артефактов других типов. Далее в разд. 3 описаны примеры удаления кардиоритмов и артефактов мышечной активности, однако этот список может быть расширен. Разработанный алгоритм может удалять и другие типы артефактов при условии, что данные артефакты обла- 

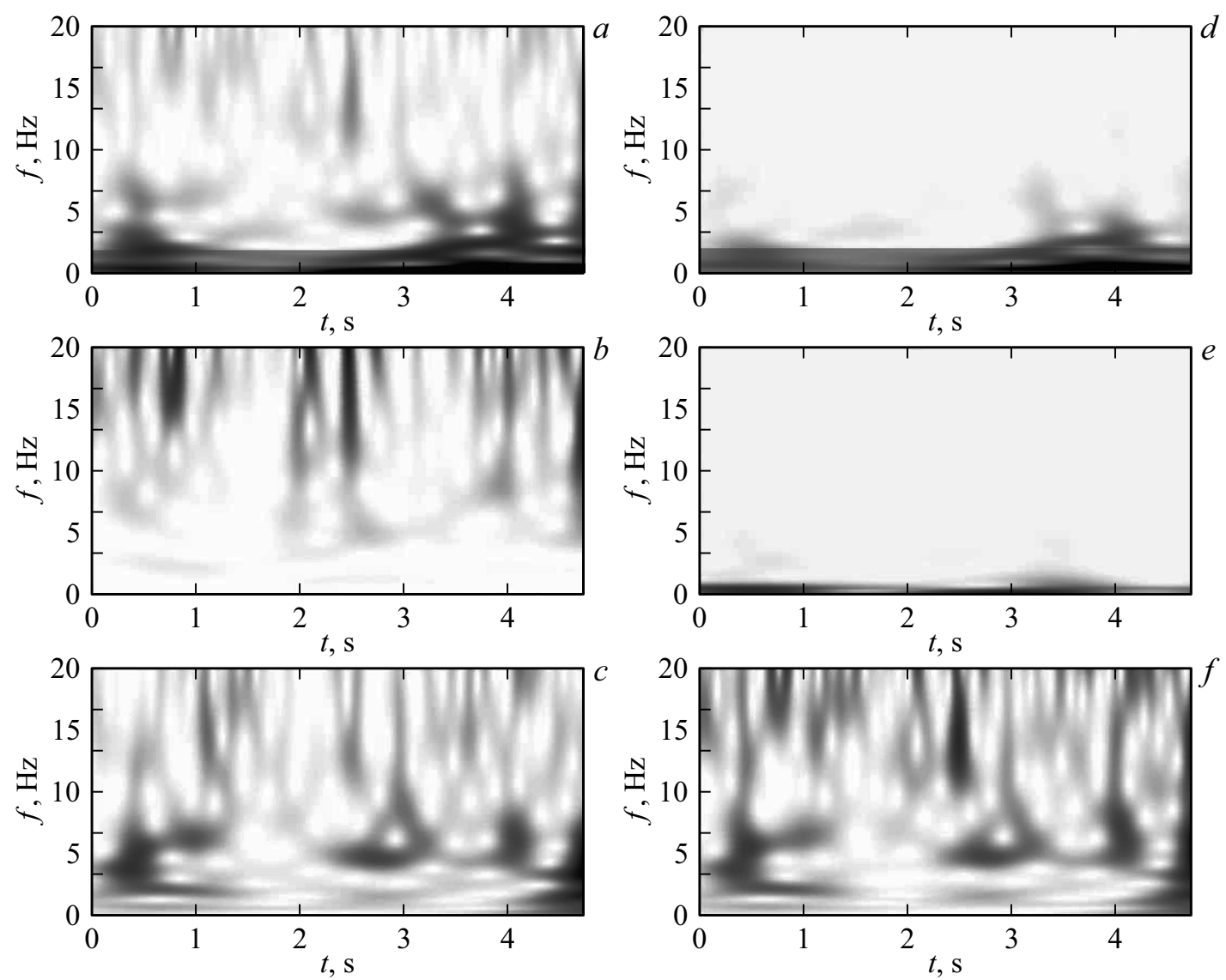

Рис. 5. Пример работы метода для фильтрации сигналов ЭЭГ от артефактов: вейвлетные спектры, рассчитанные для сигнала ЭЭГ с несколькими глазодвигательными артефактами (образ артефактов на спектре выделен затененной рамкой) (a), четырех эмпирических мод сигнала ЭЭГ $(b-e)$ и восстановленного сигнала ЭЭГ $(f)$.

дают достаточно выраженным образом на вейвлетном спектре и соответствующие им эмпирические моды могут быть идентифицированы.

\section{3. Результаты}

Разработанный метод был протестирован на примере удаления физиологических артефактов нескольких типов с экспериментальных сигналов ЭЭГ человека. Сигналы ЭЭГ были записаны с использованием электроэнцефаллографа „Энцефалан-19/26“ (Медиком-МТД, Таганрог, Россия) при помощи стандартной схемы расстановки электродов - Международной системы „10-20“ [28]. Сигналы были отфильтрованы регистрирующей аппаратурой в диапазоне частот $0.016-70 \mathrm{~Hz}$ с полосовым фильтром в районе $49.5-50.5 \mathrm{~Hz}$ для устранения влияния промышленной электрической сети. Дизайн экспериментов включал в себя стандартные физиологические пробы. Все эксперименты были проведены для 15 условно здоровых мужчин и женщин в возрасте 18-40 лет. Все эксперименты были одобрены этическим комитетом Саратовского государственного технического университета им. Ю.А.Гагарина.
В ходе проведения экспериментов на записях ЭЭГ было обнаружено несколько типов артефактов: глазодвигательные, кардиоритмы, мышечные, каждый из которых встречается в диапазоне частот $0.5-15 \mathrm{~Hz}$, что пересекается с диапазоном информативных паттернов на сигналах ЭЭГ, следовательно, фильтрация данных артефактов является важной задачей.

Результаты работы предложенного метода представлены на рис. 6, на котором приведены экспериментальные сигналы ЭЭГ человека, содержащие глазодвигательные артефакты (рис. 6, $a$ ), кардиоритмы (рис. 6, $b$ ) и артефакты мышечной активности (рис. 6,c). Также в правой части рис. 6 представлены сигналы ЭЭГ после фильтрации предложенным методом. Видно, что в каждом из случаев артефакты были удалены в ходе фильтрации. Также следует отметить, что низкочастотная огибающая сигнала ЭЭГ, которая не содержит полезной информации, также была удалена после применения разработанного метода. Таким образом, предложенный метод может быть использован не только для удаления различных типов физиологических артефактов на ЭЭГ, но также для фильтрации некоторых шумовых компонент.

Эффективность разработанного метода была продемонстрирована на примере удаления глазодвигательных артефактов с экспериментальной записи ЭЭГ человека. 


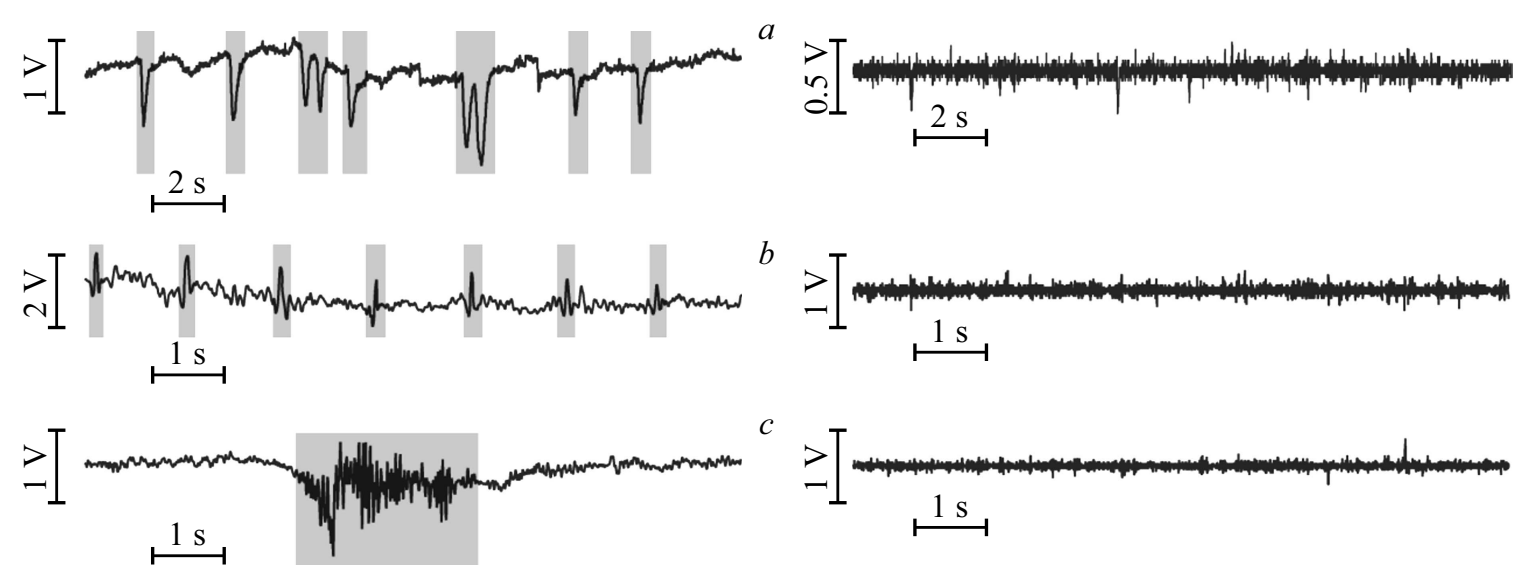

Рис. 6. Результаты фильтрации сигнала ЭЭГ от артефактов трех типов: глазодвигательных $(a)$, кардиоритмов $(b)$, мышечной активности $(c)$; сигналы ЭЭГ до фильтрации приведены в левой части рисунка, сигналы после фильтрации - в правой; артефакты обозначены затененными рамками.

Была рассмотрена запись продолжительностью $600 \mathrm{~s}$, на которой было зарегистрировано 95 артефактов с амплитудой от 1 до $4 \mathrm{~V}$. Критерием удаления артефакта было снижение его амплитуды после фильтрации до уровня средней амплитуды сигнала ЭЭГ. Средняя амплитуда ЭЭГ считалась исходя из амплитуды фоновой активности и различных информативных паттернов, но не артефактов; средняя амплитуда ЭЭГ в данном случае составила $0.6 \mathrm{~V}$. В ходе фильтрации с записи ЭЭГ было удалено 88 артефактов, и точность разработанного метода составила $\sim 92 \%$. Аналогичные результаты были получены и для остальных испытуемых. Кроме того, у артефактов, которые не были удалены полностью, была значительно снижена амплитуда (снижение составляло вплоть до $70 \%$ от исходной амплитуды), что также полезно для фильтрации сигнала ЭЭГ.

Также при анализе эффективности метода была рассчитана количественная характеристика искажения спектра сигнала до и после фильтрации разработанным методом. Для этого были рассчитаны вейвлетные спектры в частотном диапазоне $\Delta f=5-15 \mathrm{~Hz}$ для исходного и фильтрованного сигналов ЭЭГ. Затем количественная характеристика искажения была рассчитана как:

$$
M=\frac{1}{E} \int_{\Delta f} \int_{0}^{\tau}\left|W(f, t)-W_{E M}(f, t)\right| d t d f,
$$

где $W(f, t)$ - амплитуда вейвлетного спектра сигнала ЭЭГ до фильтрации, $W_{E M}(f, t)$ - амплитуда вейвлетного спектра сигнала ЭЭГ после фильтрации разработанным методом, $\tau$ - длина сигнала ЭЭГ, нормировка осуществлялась на величину усредненной амплитуды вейвлетного спектра исходного сигнала

$$
E=\int_{\Delta f} \int_{0}^{\tau}|W(f, t)| d t d f .
$$

В результате расчетов было получено $M<10^{-2}$, а следовательно, искажения сигнала ЭЭГ, вызванные проце- дурой разложения по эмпирическим модам и удалением артефактов, можно считать незначительными.

\section{Заключение}

Таким образом, в настоящей работе был предложен новый метод для фильтрации сигналов ЭЭГ и удаления физиологических артефактов различных типов. Был разработан универсальный алгоритм метода, основанный на разложении сигнала по эмпирическим модам. Были рассмотрены особенности метода, включающие процедуры отбора эмпирических мод для рассмотрения и поиска мод с физиологическими артефактами с использованием непрерывного вейвлетного преобразования. Было показано, что разработанный алгоритм является универсальным для удаления артефактов различных типов. Работа предложенного метода была рассмотрена на примере удаления артефактов трех типов: глазодвигательных, кардиоритмов и мышечной активности. Метод показал высокую точность $(\sim 90 \%)$. Также был проведен расчет количественной характеристики искажения сигнала, в результате чего было показано, что искажения, вносимые в сигнал при удалении с него физиологических артефактов, являются незначительными.

Работа выполнена при поддержке РФФИ (грант 16-29-08221 офи_м), гранта президента РФ (проект МК-3305.2017.2) и Министерства образования и науки РФ (проект 3.861.2017/4.6). Экспериментальные исследования метода удаления глазодвигательных артефактов были поддержаны грантом РНФ № 16-12-10100.

\section{Список литературы}

[1] Nunez P.L., Srinivasan R. Electric fields of the brain: the neurophysics of EEG. Oxford University Press., 2006. 640 p.

[2] Божсокин C.В. // ЖТФ. 2010. Т. 80. Вып. 9. С. 16-24.

[3] Buzsaki G., Draguhn A. // Science. 2004. Vol. 304. P. 19261929. 
[4] Van Luijtelaar G., Lüttjohann A., Makarov V.V., Maksimenko V.A., Koronovskii A.A., Hramov A.E. // J. Neuroscience Methods. 2016. Vol. 260. P. 144-158.

[5] Hramov A.E., Koronovskii A.A., Makarov V.A., Pavlov A.N., Sitnikova E.Yu. Wavelets in Neuroscience. Heidelberg N.Y.-Dordrecht-London, 2015. 318 p.

[6] Павлов А.Н., Храмов А.Е., Короновский А.А., Ситникова Е.Ю., Макаров В.А., Овчинников А.А. // УФН. 2012. Т. 182. № 9. С. 905-939.

[7] Божскин С.В., Суслова И.М. // ЖТФ. 2013. Т. 83. Вып. 12. C. 26.

[8] Ebersole J.S., Husain A.M., Nordli D.R. Current Practice of Clinical Electroencephalography. Wolters Kluwer, 2014. $664 \mathrm{p}$.

[9] Luders H., Noachtar S. Atlas and Classification of Electroencephalography. Philadelphia: WB Saunders Co., 2000. 208 p.

[10] Zhang C., Tong L., Zeng Y. et al. // Biomed Res Int. 2015. P. 720450 .

[11] Uriguen J.A., Garcia-Zapirain B. // J. Neural Eng. 2015. Vol. 12. N 3. P. 031001.

[12] Меринов П.А., Беляев М.Г. // Влияние автоматических методов очистки ЭЭГ от артефактов на точность классификации - 2015: Сб. тр. 39-й Междисциплин. школыконференции ИППИ РАН „Информационные технологии и системы 2015“. М.: Изд-во Института проблем передачи информации им. А.А. Харкевича РАН, 2015. С. 313-328.

[13] Bell A.J., Sejnowski T.J. // Neural Comput. 1995. Vol. 7. N 6. P. 1129.

[14] Joyce C.A., Gorodnitsky I.F., Kutas M. // Psychophysiology. 2004. Vol. 41. N 2. P. 313-325.

[15] Gratton G. // Instruments \& Computers. 1998. Vol. 30. P. 4453.

[16] Короновский А.А., Храмов А.Е., Москаленко О.И., Грубов В.В. Патент на изобретение RUS N 2560388 от 21.07.2015.

[17] Huang N.E., Shen Z., Long S.R. et al. // Proc. R. Soc. A. 1998. Vol. 454. P. 903.

[18] Павлов А.Н., Филатова А.Е., Храмов А.Е. // Радиотехника и электроника. 2011. Т. 56. № 9. С. 1099-1106.

[19] Lindsen J.P., Bhattacharya J. // Physiology. 2010. Vol. 47. N 5. P. $955-960$.

[20] Yeh C.L., Chang H.C., Wu C.H., Lee P.L. // BioMedical Engineer. OnLine. 2010. Vol. 9. P. 25.

[21] Flandrin P., Goncalvés P. // Int. J. Wavelets Multiresolut. Inform. Process. 2004. Vol. 2. N 4. P. 477.

[22] Павлов А.Н., Филатова А.Е. // Известия вузов. ПНД. 2011. T. 19. № 1. C. 3-13.

[23] Martis R.J., Acharya R.U., Tan J.H. et al. // Intern. J. Neural Systems. 2012. Vol. 22. N 6. P. 1250027.

[24] Grubov V.V., Sitnikova E.Yu., Pavlov A.N. et al. // Proc. SPIE. 2015. Vol. 9448. P. 94481Q.

[25] Grubov V.V., Sitnikova E.Yu., Pavlov A.N., Koronovskii A.A., Hramov A.E. // Physica A. 2017. N 486. P. 206-217

[26] Guger C., Ramoser H., Pfurtscheller G. // IEEE Trans. Rehab. Eng. 2000. Vol. 8. P. 562.

[27] Guger C., Edlinger G., Harkam W., Niedermayer I., Pfurtscheller G. // IEEE Trans. Rehab. Eng. 2003. Vol. 11. P. 145.

[28] Nuwer M.R., Comi C., Emerson R. et al. // Electroencephalogr. Clin. Neurophysiol. 1998. Vol. 106. P. 259-261. 